\title{
Virtual meetings promise to eliminate geographical and administrative barriers and increase accessibility, diversity and inclusivity
}

Conference organizers must adopt a novel, comprehensive approach to ensure increased accessibility, diversity and inclusivity of post-pandemic conferences.

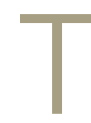

he world is facing unprecedented challenges owing to the COVID-19 pandemic. In the scientific setting, one important restriction is the lack of in-person interpersonal interactions, which form the core of academic conferences. Despite this, it is essential to continue sharing scientific knowledge. The research community was quick to adapt to COVID-19 restrictions, with the majority of conferences effectively adopting a virtual format. Online platforms provide a viable solution to the problem of sharing knowledge remotely and enable virtual connections between scientists. Sharing code, data and comments through these platforms has become easier ${ }^{1}$.

Additionally, online conferences help alleviate environmental challenges. Before the COVID-19 pandemic, there were open discussions about the substantial carbon footprint associated with conferences, attributed mainly to air travel related to in-person attendance, which contributes to human-induced climate change ${ }^{2}$. Holding conferences online means less travel, thus reducing the carbon footprint ${ }^{3}$. The advancement of technology over the past decade and the ability to attend a virtual conference from any device without the need for custom hardware ${ }^{4}$ also added to the appeal of a virtual setup.

With these benefits in mind, as well as the convenience of attending global conferences from any location, many members of the scientific community had been pushing for scientific meetings to be conducted at least in a hybrid mannerpartly in person and partly online-if not fully online ${ }^{5}$. However, this idea had not been widely adopted, perhaps due to the substantial logistical burden associated with ensuring proper internet access and organizing time zones and an unwillingness to let go of the status quo. Nonetheless, out of necessity, conferences have now developed their own virtual platforms within a short time to accommodate the present challenges, often with support from the tech industry.

There are also logistic aspects to consider. In some respects, more effort is required to put together a virtual conference than an in-person one, especially in terms of engaging prospective participants and garnering their interest for upcoming years. However, whereas in-person conferences are restricted in the number of attendees they can accommodate, virtual conferences have the major advantage that the number of participants attending, and the geographical regions the conference can reach, can be scaled up relatively easily. This not only provides flexibility in regard to who can attend but also promises to break logistical barriers associated with physical travel and to connect researchers across the globe. Virtual conferences are not bound to one physical location, which promises to increase global participation and promote inclusivity among attendees ${ }^{6,7}$. Another major advantage is the reduction of cost, not only in terms of registration fees and travel by the attendees but also in terms of organizing the conference itself ${ }^{8}$. Here, we set out to test this hypothesis by analyzing the demographics of attendees at four major conferences before and after the fully virtual format was adopted.

\section{Virtual platforms enable increased participation, diversity and inclusion} What distinguishes a conference from a series of webinars is the active participation by the attendees ${ }^{9}$. We performed a
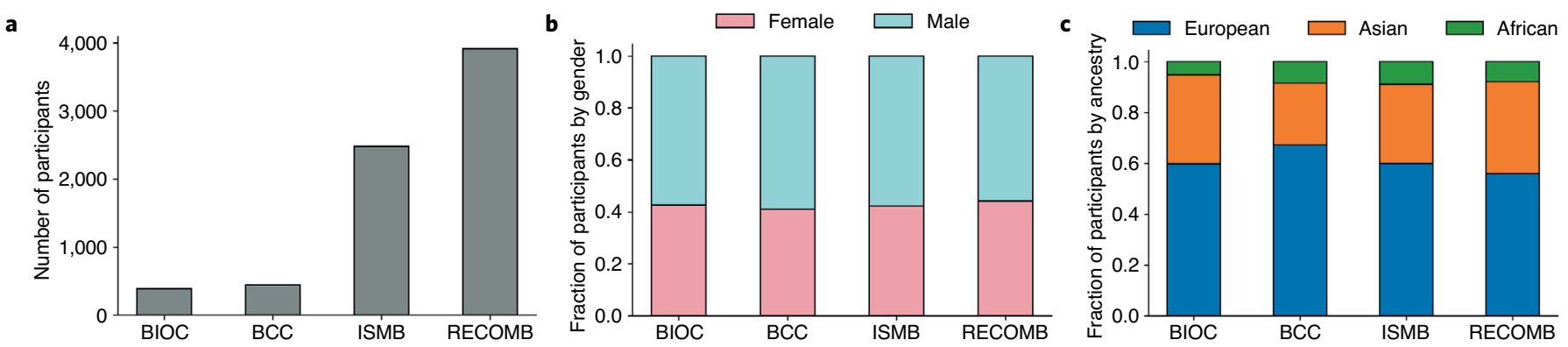

Fig. 1| Distribution of participants by gender and ethnicity for the four virtual-based conferences in 2020. $\mathbf{a}$, Total numbers of participants. $\mathbf{b}$, Percentages of females and males. c, Numbers of participants by ethnicity. BCC, Bioinformatics Community Conference; BIOC, BioConductor Annual Meeting; ISMB, Intelligent Systems for Molecular Biology; RECOMB, Research in Computational Molecular Biology. African ethnicity includes Africans, Muslim; Asian ethnicity includes East Asian, Japanese, Indian Sub Continent; and European ethnicity includes British, East European, Jewish, French, Germanic, Hispanic, Italian, Nordic, as defined in previous studies ${ }^{17}$. 

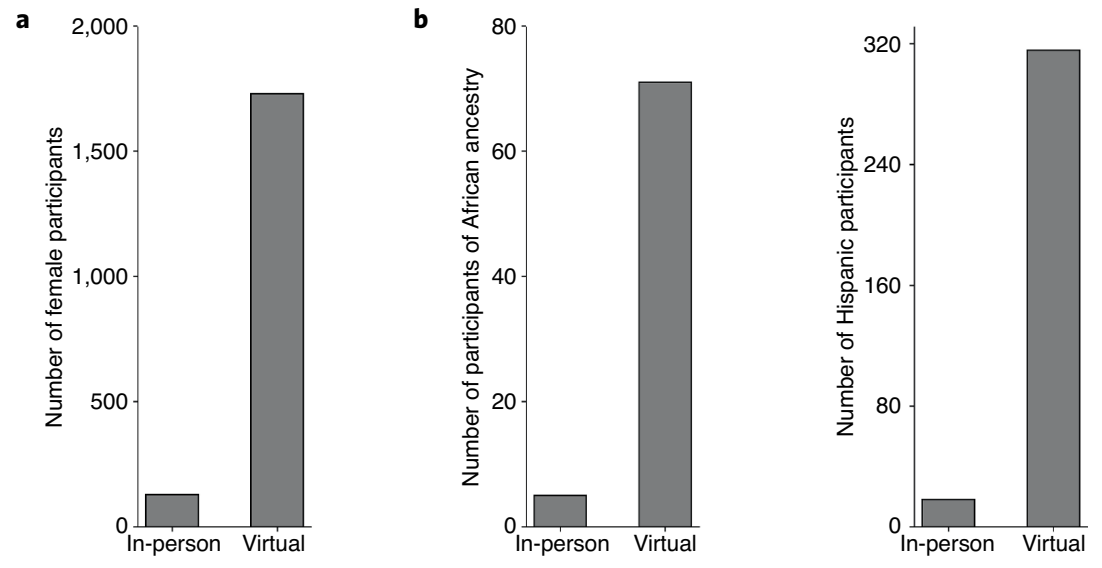

Fig. 2 | Increase in attendance by members of under-represented minority groups in $\mathbf{2 0 2 0}$ (virtual) compared to 2019 (in-person) for the RECOMB conference. a, Females. b, Africans, c, Hispanics. The number of participants is shown on the $y$ axis, and percentages are shown above each bar. Africans and Hispanics are subcategories of the African and European ethnicity groups from Fig. 1, respectively.

systematic analysis of 24 conferences between January and August 2020 across medicine, biology, computer science and other fields. Of these, 22 adapted to the virtual format, whereas 2 were canceled altogether for the year. Among the 22, we observed a decrease in registration fee overall, with most (but not all) conferences waiving the attendance fee altogether for the online conference. We selected the most popular conference, the Research in Computational Molecular Biology (RECOMB) conference, in computational biology with free registration to illustrate the impact of virtual platforms on reducing financial and administrative burdens for participants. To further illustrate the distribution of participants and speakers across gender, ethnicity and country, comparing virtual platforms in 2020 with in-person platforms in 2019, we focused on four conferences: the Bioinformatics Community Conference (BCC), BioConductor Annual Meeting (BIOC), Intelligent Systems for Molecular Biology (ISMB) and Research in Computational Molecular Biology (RECOMB) (Fig. 1 and Supplementary Table 1).

To investigate the impact of virtual conferences in more depth, we compared the distribution of participants from the in-person versus virtual platforms for one of the four conferences (RECOMB) using data from 2019 and 2020. The gender and ethnicity of participants were imputed from participant names using machine-learning approaches (see Supplementary Methods). The total number of participants rose from 374 in 2019 to 3,913 in 2020, a $900 \%$ increase, while the percentage of female participants remained the same (Fig. 2). The number of individuals belonging to under-represented minorities ${ }^{10}$ (specifically, African American and Hispanics) increased from 19 to 331 , demonstrating a substantial increase in the virtual platform reaching a broader range of under-represented communities. However, the relative proportion of attendees from under-represented minorities remains low $(6.15 \%$ (23/374) in 2019 and $9.89 \%$ (387/3913) in 2020) (Fig. 2), which may indicate that additional obstacles impede the participation of those groups in academia that were not improved after removal of financial (for example, by free registration) and administrative barriers (for example, by lack of traveling requirement).

Accompanying the overall increase of participants in the RECOMB conference, the geographical distribution increased from 19 to 73 countries across the world, including a substantial increase in participants from developing countries-including those in Africa, South America, Asia and Oceania, and 3 low-income and 35 middle-income countries (Figs. 3 and 4, and Supplementary Fig. 5). The number of participants in the virtual RECOMB conference in 2020 was considerably higher than the number who joined the in-person RECOMB conference in 2019 (Fig. 4). No participants from Oceania attended the in-person RECOMB conference in 2019, whereas 55 participants from the region attended the virtual RECOMB conference in 2020. A similar trend is also observed among participants from African countries (Figs. 3 and 4, and Supplementary Fig. 5). Collectively, these results demonstrate an increased diversity of participants due to the use of virtual as compared to in-person platforms.

\section{Virtual platforms reduce engagement in social media interactions}

Inspired by the increased number of participants from virtual conferences, we explored the trend of online social activity as a proxy for measuring the engagement of participants in the conference activities. We hypothesized that a greater number of participants might lead to increased engagement in social media. However, this was not the case. Among three select large conferences of more than 1,000 participants from computational biology, bioinformatics and medical oncology (American Society of Clinical Oncology (ASCO), the American Association for Cancer Research (AACR), ISMB and BIOC), we quantified the number of tweets three days before, during and three days after the dates of each conference for the past five to ten years (ASCO 2010-2020, Supplementary Fig. 1; AACR 2015-2021, Supplementary Fig. 2; ISMB 2015-2020, Supplementary Fig. 3; BIOC 2015-2020, Supplementary Fig. 4). For AACR, we collected the new data from 2021, as its conference had already occurred (April 2021) at the time of this study. We used hashtags to retrieve tweets relevant to each conference on Twitter (Supplementary Table 2). We observed a continuing growth in related tweets from the earliest time period until they reached a potential peak on the first or second day of the conference. Comparing 2019 and 2020, we observed an overall decline of tweets in 2020 for ASCO, AACR and ISMB (Supplementary Figs. 1-3), while those for BIOC remained similar (Supplementary Fig. 4).

\section{Discussion}

As a result of the ongoing pandemic, we have witnessed the evolution from a world where in-person meetings were the norm to a new reality in which nearly all conferences in the last year have been held virtually. There are positive aspects of this new format that support its continuation going forward. Online conferences increase participation from scientists belonging to under-represented groups and from developing countries, and offer advantages such as schedule flexibility, reduced registration fees and removal of travel barriers. Accordingly, in our analyses, we observed a dramatic 
a

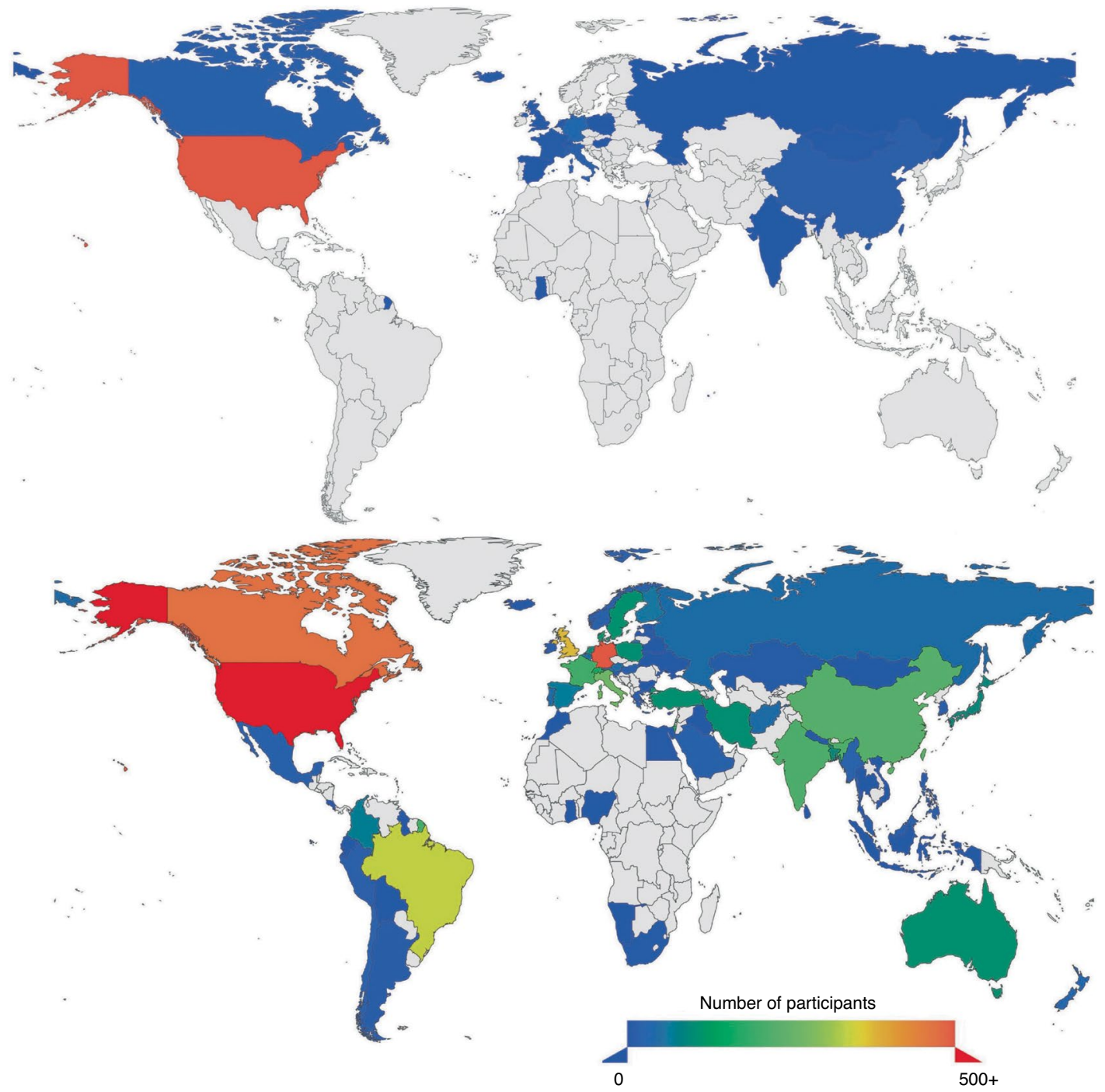

Fig. 3 | Increased diversity and inclusion of participants in the RECOMB conference in virtual compared to in-person formats. $\mathbf{a}$, $\mathbf{b}$, Geographic distribution of the affiliations of participants in 2019 (a) and 2020 (b). Color represents the number of participants from each country (blue to red: 0 to $>200$ ).

increase in the number of participants from under-represented minorities, as well as in international attendees, including those from developing countries. The virtual conference platform offers increased accessibility, reduced administrative burden and more flexible programs compared to in-person meetings, especially for international participants ${ }^{7}$. The remote format allowed more people to present their work and attend events by offering recorded lectures by on-demand online services and eliminating physical space limitations. Many conferences now provide recorded videos and workshop materials for an extended time after the conference ends, and even post some of these materials to public media channels such as YouTube, open to the public permanently. In addition, select conferences such as the Keystone
Symposia have published all recorded talks online free of charge to its members from developing countries, removing financial, administrative and geographic barriers. An open-science environment has also been promoted through Slack channels for conference reactions, conversations and comments. The advantage of these online communication channels is that they are free, long-term and sustained for as long as participants have the need.

One important feature of virtual conferences, which has not been available in in-person meetings, is live captions on screen, along with Computer Aided Real-Time Transcription (CART) and American Sign Language (ASL) interpretations. Captions in real time provide higher accessibility to participants with disabilities and non-native English speakers and enhance participants' attention and accurate understanding of the content covered by the speaker. However, we must note that live captioning could be an added expense and might not be an option if conferences are to be kept affordable. Delayed crowd-sourcing has been considered as an option by some conferences to provide such a service. Including a communicator for the hearing impaired is recommended and has been implemented in select conferences and online seminars, improving accessibility for all participants. In addition, conferences often deposit recorded talks to online video platforms. One example is the BIOC conference, where automatic creation of captions is available via speech recognition technology. This is dramatically beneficial and should be considered for future conferences. 


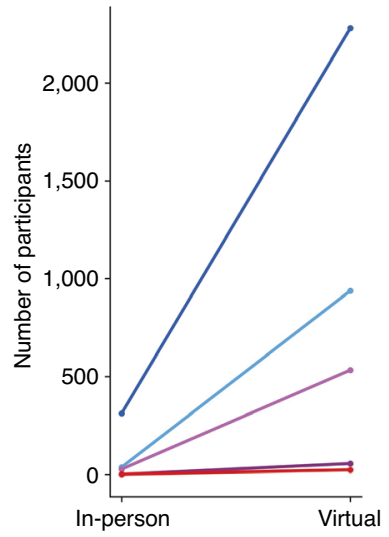

b

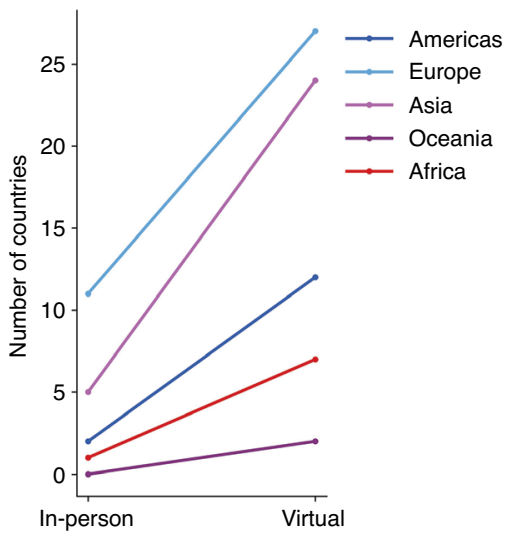

Fig: 4 | Comparison of the number of participants and countries per continent in virtual and in-person formats of the RECOMB conference. $\mathbf{a}$, Comparison of the absolute numbers of participants who attended the RECOMB conference in each continent in the virtual and in-person format. $\mathbf{b}$, The absolute numbers of countries with at least one participant attending the virtual and in-person format of the RECOMB conference across different continents.

However, this change has not been without its difficulties. We have witnessed new challenges associated with the virtual conference platform, including requirements for high-speed internet, reduced peer interactions, 'screen fatigue ${ }^{11}$ from the need to spend substantial periods of time in front of a computer, interference from work and home responsibilities, lack of the social interaction $^{12}$ that would be possible in an in-person setting, and the complications of connecting across time zones. Further, there are two significant drawbacks of virtual conferences: first, the reduced opportunity for collaboration and networking between specialties; and second, the limited networking opportunities of casual interactions in poster halls or social events, which can lead to new collaborations, awareness of new findings and career advancement. This implies that in-person conferences will always have an advantage with respect to these aspects, and when we conceptualize the hybrid conference format going ahead, we need to ensure that they are maintained. Disability, visa requirements, travel time and cost, and inflexible schedules are among the barriers that prevent researchers from under-represented groups and developing countries from participating in in-person scientific and medical conferences internationally ${ }^{13}$. Therefore, we believe that organizers should continue striving to provide travel fellowships and services $^{7}$ (such as childcare and disability services) to allow broad access to the in-person experience.

Although virtual conferences reduce costs for attendees, the financial considerations for organizers are also a significant issue. Academic conferences are typically organized by professional societies (for example, the AACR, the International Society for Computational Biology (ISCB) and so on) and contribute significantly to the societies' revenue and membership, which in turn benefits the societies' mission, programs and initiatives such as lobbying for research funding, developing statements and standards, promoting and delivering education to its members, organizing outreach events and providing travel and other types of fellowships. On one hand, as compared to in-person events, virtual conferences result in cost savings in food and beverage costs, poster board costs and rental fees. Audiovisual costs, however, are comparable between in-person and virtual events, and virtual events add logistical complexity and require more time from organizers, technicians and presenters for rehearsals and for capturing, uploading and quality control of videos. A further downside for organizers is that virtual events may not drive membership in the same way as in-person events. For example, ISCB memberships decreased in 2020 compared with 2019 and do not appear to be on track to recover in 2021. Furthermore, some of the costs and revenues from academic conferences are often covered by sponsorships from industry and other organizations. It has been difficult to convince sponsors that they will receive equal return on investment in virtual conferences, and when sponsors do participate, their contributions are smaller. When this is combined with a reduction in registration fees, virtual conferences yield lower overall revenues for organizers than in-person meetings.

We noted that despite the increase in the number of attendees at virtual conferences, social media engagement during those conferences did not increase as the number of participants did (Supplementary Figs. 1-4). However, we acknowledge that our method might not reflect all social media activity from those conferences, given some also have their own communication platforms outside of Twitter. One possible explanation for such a phenomenon is that unlike in-person conferences, some virtual conferences might use different ways for participants to communicate besides social media. Additionally, attendees at in-person conferences might have more engagement in the live sessions, be less likely to experience technology fatigue and, conversely, be more likely to share their experience and thoughts on social media compared to attendees of virtual conferences. We also acknowledge that our Twitter data do not normalize for increasing usage over time. In addition, different time zones may present logistic challenges for participants from other countries. Although we observed a higher number of participants in virtual conferences, those active in social media may be fewer than those at in-person conferences, potentially due to challenges such as the difficulty of completely separating day-to-day work or family-related duties from participation in a virtual conference online, which could prevent participants (especially caregivers) from being fully engaged in the conference. Thus, it can be assumed that even though participation has increased drastically, this does not necessarily mean that all attendees engage with the conference to a maximum extent. Given the convenience of attending the conference from home, participants may concentrate only on specific talks that garner their interest, rather than attending as many talks as possible as is common at in-person conferences. Therefore, virtual conferences, compared to in-person conferences, may draw a larger group of attendees that includes people who are less fully engaged.

Our findings provide evidence favoring a hybrid format for future conferences, marrying the strengths of both in-person and virtual platforms. This would broaden 
conferences' reach to more communities and a higher number of countries. Going forward, we advocate a hybrid mode of organizing conferences. Although we strongly believe that in-person conferences have their own benefits, and that no online communication tool can mimic the in-person experience completely ${ }^{14}$, we cannot neglect the multiple advantages that online conferences offer: in addition to providing opportunities to previously under-represented groups to attend global conferences, use of a hybrid format will contribute toward decarbonizing conference travel after the pandemic $\mathrm{c}^{3,15,16}$. In fact, several conferences have started to implement such a hybrid mode. The Medical Image Computing and Computing Assisted Intervention Society (MICCAI) announced both in-person and virtual plans, though it ultimately had to cancel the former due to uncertainties of the pandemic. The Society for Immunotherapy of Cancer, the Society for Melanoma Research (SMR), the Cold Spring Harbor Laboratory (CSHL) Genome Informatics, the Basel Computational Biology Conference (BC2) and AGBT (Advances in Genome Biology and Technology) Precision Medicine have all announced hybrid formats for their meetings. These stand as evidence for the growing need for and acceptance of the hybrid conference format. Taken together, the results of our study warrant the ongoing evaluation on data from future conferences with different platforms (in-person, virtual or hybrid) to assess its influence on accessibility, inclusion and diversity.

\section{Data availability}

All data required to produce the figures and analysis performed in this paper are freely available at https://github.com/ HCC-data-sciences-pub/virtualconference-analysis.

\section{Code availability}

All code required to produce the figures and analysis performed in this paper is freely available at https://github.com/ HCC-data-sciences-pub/virtual-conferenceanalysis.

\section{Juncheng Wu (D) 1,10, Anushka Rajesh (D) 2,10, Yu-Ning Huang (D) 3,10, Karishma Chhugani (D)2, Rajesh Acharya (D) 4 , Kerui Peng (D) 3 , \\ Ruth D. Johnson ${ }^{5}$, Andrada Fiscutean ${ }^{6}$, Carla Daniela Robles-Espinoza (D) 7, Francisco M. De La Vega (D) 8 , Riyue Bao (D) 4,9 and Serghei Mangul (D) ${ }^{3 \times}$ ${ }^{1}$ Allegheny College, Meadville, PA, USA. ${ }^{2}$ Department of Pharmacology \& Pharmaceutical Sciences, University of Southern California School of Pharmacy, Los Angeles, CA, USA. ${ }^{3}$ Department of Clinical Pharmacy, University of Southern California School of Pharmacy, Los Angeles, CA, USA. ${ }^{4}$ UPMC Hillman Cancer Center, Pittsburgh, PA, USA. ${ }^{5}$ Department of Computer Science, University of California, Los Angeles, Los Angeles, CA, USA. ${ }^{6}$ Faculty of Journalism and Communication Studies, University of Bucharest, Bucharest, Romania. ${ }^{7}$ International Laboratory for Human Genome Research, National Autonomous University of Mexico, Santiago de Querétaro, Mexico. ${ }^{8}$ Department of Biomedical Data Science, Stanford University School of Medicine, Stanford, CA, USA. ${ }^{9}$ Department of Medicine, University of Pittsburgh, Pittsburgh, PA, USA. ${ }^{10}$ These authors contributed equally: Juncheng Wu, Anushka Rajesh, Yu-Ning Huang. \\ 凶e-mail:baor@upmc.edu; serghei.mangul@gmail.com}

Published online: 29 December 2021 https://doi.org/10.1038/s41587-021-01176-Z
References

1. Sarabipour, S. eLife 9, e62668 (2020)

2. Johnson, R., Fiscutean, A. \& Mangul, S. Preprint at https://arxiv.org/abs/2002.12268 (2020).

3. Barral, A. Nat. Ecol. Evol. 4, 666-667 (2020).

4. Welch, C. J., Ray, S., Melendez, J., Fare, T. \& Leach, M. Nat. Chem. 2, 148-152 (2010).

5. Newman, C. J. Dev. Med. Child Neurol. 63, 493 (2021).

6. Levitis, E. et al. Preprint at PsyArXiv https://doi.org/10.31234/osf. io/vj5tu (2021).

7. Sarabipour, S. et al. Nat. Hum. Behav. 5, 296-300 (2021).

8. Achakulvisut, T. et al. Trends Cogn. Sci. 25, 265-268 (2021).

9. Roos, G., Oláh, J., Ingle, R., Kobayashi, R. \& Feldt, M. Comput Theor. Chem. 1189, 112975 (2020).

10. National Center for Science and Engineering Statistics, Directorate for Social, Behavioral and Economic Sciences, National Science Foundation. Women, Minorities, and Persons with Disabilities in Science and Engineering 2021 (NCSES, 2021); https://ncses.nsf.gov/pubs/nsf21321/ report/persons-with-disability

11. Foramitti, J., Drews, S., Klein, F. \& Konc, T. J. Clean. Prod. 294, 126287 (2021).

12. Achakulvisut, T. et al. eLife 9, e57892 (2020).

13. Pacchioni, G. Nat. Rev. Mater. 5, 167-168 (2020).

14. Ahn, S. J. et al. Front. Virtual Real. 2, 648575 (2021).

15. Klöwer, M., Hopkins, D., Allen, M. \& Higham, J. Nature 583, 356-359 (2020)

16. Gibson, H., Illingworth, S. \& Buiter, S. Geosci. Commun. 4, 437-451 (2021).

17. Ambekar, A., Ward, C., Mohammed, J., Male, S. \& Skiena, S. in Proc. 15th ACM SIGKDD International Conference on Knowledge Discovery and Data Mining 49-58 (Association for Computing Machinery, 2009); https://doi.org/ $10.1145 / 1557019.1557032$

Acknowledgements

The authors thank M. Alekseyev for his assistance with data inquiry and D. Kovats of the ISCB for responding to our queries. S.M. and K.P. are supported by US National Science Foundation grants 2041984 and 2135954. R.B. acknowledges funding from the Hillman Fellows for Innovative Cancer Research Program.

Competing interests

The authors declare no competing interests.

\section{Additional information}

Supplementary information The online version contains supplementary material available at https://doi.org/10.1038/s41587-021-01176-z. 